\title{
Correction to: Auditory and frontal anatomic correlates of pitch discrimination in musicians, non-musicians, and children without musical training
}

\author{
María-Ángeles Palomar-García ${ }^{1}(1) \cdot$ Mireia Hernández ${ }^{2} \cdot$ Gustau Olcina $^{3}$. Jesús Adrián-Ventura ${ }^{1}$ - Víctor Costumero ${ }^{4}$. \\ Anna Miró-Padilla ${ }^{1}$ Esteban Villar-Rodríguez ${ }^{1}$. César Ávila ${ }^{1}$
}

Published online: 31 October 2020

(c) Springer-Verlag GmbH Germany, part of Springer Nature 2020

\section{Correction to: Brain Structure and Function https://doi.org/10.1007/s00429-020-02151-1}

In the original version of the article, the abstract section was missed to be added, and the abstract should read as below:

\begin{abstract}
Individual differences in pitch discrimination have been associated with the volume of both the bilateral Heschl's gyrus and the right inferior frontal gyrus (IFG). However, most of these studies used samples composed of individuals with different amounts of musical training. Here, we investigated the relationship between pitch discrimination and individual differences in the gray matter (GM) volume of these brain structures in 32 adult musicians, 28 adult non-musicians, and 32 children without musical training. The results showed that (1) the individuals without musical
\end{abstract}

training (whether children or adults) who were better at pitch discrimination had greater volume of auditory regions, whereas (2) musicians with better pitch discrimination had greater volume of the IFG. These results suggest that the relationship between pitch discrimination and the volume of auditory regions is innately established early in life, and that musical training modulates the volume of the IFG, probably improving audio-motor connectivity. This is the first study to detect a relationship between pitch discrimination ability and GM volume before beginning any musical training in children and adults.

The original article has been corrected.

The original article can be found online at https://doi.org/10.1007/ s00429-020-02151-1.

María-Ángeles Palomar-García mpalomar@uji.es

1 Neuropsychology and Functional Neuroimaging Group, Department of Basic Psychology, Clinical Psychology and Psychobiology, Universitat Jaume I, Avda. Sos Baynat, s/n., 12071 Castellón de la Plana, Spain

2 Cognition and Brain Plasticity Group, Department of Cognition, Development and Educational Psychology, Institut de Neurociències, University of Barcelona, Barcelona, Spain

3 Neuropsychology and Functional Neuroimaging Group, Department of Education, University Jaume I, 12071 Castellón, Spain

4 Center for Brain and Cognition, University Pompeu Fabra, Barcelona, Spain 